\title{
The Color of Money: Threshold Effects in Quantum Economics
}

\author{
David Orrell (1)
}

check for

updates

Citation: Orrell, D. The Color of

Money: Threshold Effects in

Quantum Economics. Quantum Rep

2021, 3, 325-332. https://doi.org/

10.3390 /quantum 3020020

Received: 30 March 2021

Accepted: 12 May 2021

Published: 14 May 2021

Publisher's Note: MDPI stays neutral with regard to jurisdictional claims in published maps and institutional affiliations.
Systems Forecasting, Toronto, ON M5R 2V9, Canada; dorrell@systemsforecasting.com

\begin{abstract}
Many cognitive phenomena of the sort studied by behavioral psychologists show evidence of a threshold effect, where a certain minimum impulse is required in order to produce a change. An example is the phenomenon of preference reversal, where a change in context affects a decision, but only if the effect on perceived utility is sufficiently large. Similar threshold effects play a role in the endowment effect, where the change of context from owning to buying something induces a step change in its perceived value, or the ultimatum game, where people demand a certain minimum threshold amount before a deal can be accepted. The situation is similar to the photoelectric experiment in physics, where a minimum threshold of energy from a photon is required in order to dislodge an electron from an atom. In physics, this quantum of energy is written as the product of Planck's constant and frequency. This paper uses the concept of entropic force to derive a similar expression for quantum economics. The theory is applied to a range of cognitive and economic phenomena exhibiting a threshold effect.
\end{abstract}

Keywords: decision making; quantum cognition; entropic forces; quantum economics

\section{Introduction}

Quantum physics was inspired by the observation that energy is transmitted in discrete parcels which physicists called quanta, from the Latin for "how much". The existence of quanta was first proposed by Planck in 1901 as a kind of trick to explain why the so-called ultraviolet catastrophe did not occur in practice (according to classical theory, the energy carried by radiation would become unbounded at high frequencies) [1]. The fact that quanta were more than a mathematical convenience was demonstrated by Einstein in 1905, using the photoelectric effect, where an electron must receive a certain threshold of energy in order to be dislodged from an atom [2]. As he wrote, "Energy, during the propagation of a ray of light, is not continuously distributed over steadily increasing spaces, but it consists of a finite number of energy quanta localized at points in space, moving without dividing and capable of being absorbed or generated only as entities."

Almost a century later, the field of quantum cognition [3] was inspired by the observation that many features of human cognition are amenable to a quantum approach, which naturally accounts for effects such as interference between incompatible concepts, or entanglements between parties. Researchers in quantum finance have similarly argued that problems of the sort treated in quantitative finance are best expressed using the formalism of quantum mechanics [4-7]. Quantum methods are also being adopted in other areas of the social sciences such as international relations [8-10].

Many cognitive and financial phenomena show evidence of a threshold effect, where a certain minimum impulse is required in order to produce a change, as in the photoelectric effect. Examples are preference reversal, where a change in context affects a decision; the endowment effect, where the change of context from owning to buying something induces a step change in its perceived value; or the ultimatum game, where people demand a certain minimum threshold amount before a deal can be accepted. These threshold phenomena can be understood using methods based on quantum probability, as discussed below, which leads inevitably to the notion of a quantum of action or of energy. However, while Planck (1949) described the "elementary quantum of action" (which others called 
Planck's constant) as the "absolute core" of quantum mechanics [11], the cognitive version has remained only loosely defined, with the equivalent of Planck's constant being treated as an arbitrary scaling factor to specify things such as price change [12], the "mental energy" of voters' cognitive states [13], or "the energy of a social quantum of excitation" [14].

The latter work describes a "quantum social laser" where a threshold energy is given by a version of the Planck-Einstein equation $E=\hbar v$, where the frequency $v$ refers to the frequency of communications and again points out that "We definitely cannot expect that a kind of 'social Planck constant' exists" [14]. However, while it is certainly true that there is no direct map between subatomic physics and the social world and there is no general measure of something like mental or social energy, this paper argues that, in the specific context of monetary exchange, it is possible to at least develop a reasonable and useful definition for a quantum of value, which depends on a different kind of frequency that is colloquially referred to as the color of someone's money.

The outline is as follows: Section 2 explains the entropic force argument, uses it to obtain an expression for the threshold energy and exploits a previously published model of financial transactions in order to express this energy as a multiple of a frequency. Section 3 applies the method to a range of cognitive and economic phenomena exhibiting a threshold effect. Section 4 derives an expression for the energy of a money object. Section 5 discusses the economic interpretation of the formula and Section 6 summarizes the main conclusions.

\section{The Entropic Force}

As described in previous works $[15,16]$, we first define the propensity function for a buyer or seller to be the probability distribution $P(x)$ which specifies the probability of the person buying or selling at a particular price $x$, where $x$ is usually chosen to be a logarithmic variable. The resistance to changing to some nearby price $x+\Delta x$ will depend on the change in propensity conditional on (or relative to) the current propensity. This is equal to the slope of the propensity, divided by the current propensity, or $P^{\prime}(x) / P(x)$. We therefore define the associated force as $F(x)=\gamma P^{\prime}(x) / P(x)$, where $\gamma$ is a scaling parameter with units of energy.

In statistical physics, this force is known as an entropic force, because it acts to minimize entropy $[17,18]$. In economics, the force represents the mental desire for a buyer or seller to adjust the price to their own preferred level. The work done in a change of log price from $x_{1}$ to $x_{2}$ is

$$
\Delta E=\int_{x_{1}}^{x_{2}} F(x) d x=\gamma \log \left(\frac{P\left(x_{2}\right)}{P\left(x_{1}\right)}\right)
$$

which depends only on the relative initial and final propensities (so it is not necessary to normalize the propensity function).

The most basic application of the entropic force approach is to the question of supply and demand, which underpins financial transactions in general. Reference [15] considered the case where the joint propensity function, which gives the probability of a transaction occurring between a buyer and a seller, is a scaled normal curve with mean $\mu$ and standard deviation $\sigma$. This is the case for example when the propensity curves for the buyer and seller are each normal. The entropic force is then given by the linear equation

$$
F(x)=\frac{-\gamma(x-\mu)}{\sigma^{2}}=-k(x-\mu)
$$

where $k=\gamma / \sigma^{2}$ is a force constant, which is the equation for a harmonic oscillator.

While supply and demand can be modeled as a classical oscillator, the quantum framework is well suited for studying such questions for a number of reasons. One is that the price does not behave in a classical fashion: it is indeterminate until observed through a transaction, so transactions act as a process of measurement [19] and it does not oscillate in a deterministic way like a classical oscillator. The system is also naturally quantized, in the sense that there can only be a discrete number of participants in the process and a single transaction at a time. Finally, the decision to buy or sell is a cognitive 
act that involves effects such as interference which are best handled using a quantum approach $[20,21]$. In particular, for this paper the results in the next section rely on the use of quantum probability, which allows for negative probabilities and, therefore, interference, so we require our model to be consistent with the quantum approach.

The quantized version of the supply/demand model [15] is a quantum harmonic oscillator with a mass

$$
m=\frac{\hbar}{2 \omega \sigma^{2}}
$$

and force constant

$$
k=m \omega^{2}=\frac{\hbar \omega}{2 \sigma^{2}}
$$

However, the entropic force equation gives $k=\gamma / \sigma^{2}$ from which it follows that $\gamma=\hbar \omega / 2$. The Hamiltonian of the system then reflects the restoring force, while the ground state correctly matches the joint propensity curve. Note that the propensity change between the maximum at $x_{1}=0$ and $x_{2}=\sqrt{2} \sigma$ satisfies $P\left(x_{2}\right) / P\left(x_{1}\right)=1 / e$ where $e$ is Euler's number, so the corresponding energy gap is $\Delta E=\hbar \omega / 2$. This is the same as the increase in energy attained by using the corresponding displacement operator on the quantum harmonic oscillator.

To summarise, the entropic force approach allows us to express the energy gap between two states as the logarithm of the relative change in propensity, multiplied by a scaling factor $\gamma$ which has units of energy. Comparison with a previously developed quantum model of supply and demand, which applies to financial transactions in general, leads to the expression $\gamma=\hbar \omega / 2$. The resulting quantum model reflects the non-classical nature of the system; captures both its stochastic and dynamic properties; allows us to interpret variables such as mass and frequency and is consistent with the quantum cognition approach used below.

\section{The Quantum Threshold Effect}

The idea of an energy gap can also be applied to situations where a change in the subjective appeal of a particular prospect, due to a change in context, leads to a decision reversal. One approach is to model decisions as a sequence of projections, where a quantum mental state is projected first onto a subjective context and next onto an objective decision [3]. Another approach is quantum decision theory [20] which treats subjective and objective factors in parallel through the use of entanglement, rather than sequentially, but gives the same results (in fact, the two approaches are formally identical, since any sequence of two projections can be expressed as a two-qubit circuit in which the qubits are entangled [16]).

Quantum decision theory decomposes the relative appeal of a prospect $\pi$ into two components, an objective utility $f(\pi)$ and a subjective attraction $q(\pi)$. In the quantum model, these two terms interfere with each other in a context-dependent manner. If a gamble offers a choice of two prospects $\pi_{1}$ and $\pi_{2}$ then the utility function satisfies $f\left(\pi_{1}\right)+f\left(\pi_{2}\right)=1$, as with a classical probability, while the attraction function satisfies $q\left(\pi_{1}\right)+q\left(\pi_{2}\right)=0$.

According to classical utility theory, the person is expected to choose prospect $\pi_{1}$ if $f\left(\pi_{1}\right)-f\left(\pi_{2}\right)>0$. In quantum decision theory, however, the relevant test, in order for the less subjectively attractive option to be selected, becomes $f\left(\pi_{1}\right)-f\left(\pi_{2}\right)>2\left|q\left(\pi_{1}\right)\right|$. If in the lack of information about the attraction function we assume a uniform prior for the various probabilities, it is easily seen that the utility (on a scale of 0 to 1 ) of the less subjectively attractive option should typically exceed that of the other one by $0.5 \mathrm{in}$ order to be accepted [20]. 
This condition is known as the preference reversal criterion. For the case that the less attractive option has an associated cost $x_{1}$ and the more attractive option has a cost $x_{2}$, we can then write the two-valued utility function

$$
\begin{aligned}
& f\left(x_{1}\right)=\frac{x_{2}}{x_{1}+x_{2}} \\
& f\left(x_{2}\right)=\frac{x_{1}}{x_{1}+x_{2}}
\end{aligned}
$$

which describes the normalized utilities and can be identified with the objective propensity function for the two options [22]. The preference reversal condition above is then seen to hold if $x_{2} / x_{1}>3$. Again, this ratio is close to $e$ so the energy gap is close to $\Delta E=\hbar \omega / 2$. The preference reversal criterion can therefore be viewed as a general example of the threshold effect, where the energy gap is provided by the switch in the subjective attraction function due to a change in context (so in the classical model, the threshold effect would be zero).

A related phenomenon is the endowment effect, where people assign a higher value to an object that they own and are selling, than to one that they do not own and are buying. This can be viewed as another example of preference reversal, since the context has changed from selling to buying. The effect has been illustrated in a number of experiments, the best-known being one in which subjects were given a mug and then offered the chance to sell or exchange it [23]. The experimenters found that people demanded more than twice as much in exchange for the mug (a median selling price of USD 7.12), as they were willing to spend to purchase the mug themselves (median buying price of USD 2.87). The price ratio is about 2.5, which is close to $e$.

Another example is the well-known psychological experiment called the ultimatum game [24]. Two subjects are offered an award of say ten dollars, but are given an ultimatum: one must decide how to split the money and the other has to decide whether to accept the offer. If the offer is rejected, all the money is returned, so they both lose. Standard theory, based on rational utility maximizing behavior, would imply that any offer would be accepted, no matter how low, because it is better than nothing. However, the game has been performed in many countries around the world and the results consistently show that people reject an offer that is overly cheap, with about half of all responders rejecting offers below three dollars [25]. Following the same procedure as above for this threshold gives a utility ratio of 2.33. Again, this could be viewed as a variant of preference reversal, since the context has changed from price setter to price taker.

While such cognitive effects are usually demonstrated under controlled conditions using experimental subjects, a natural experiment for preference reversal was provided by the observed rate of strategic default during the US housing crisis [26]. According to objective utility maximization, default makes sense if the costs associated with staying in a home exceed the costs associated with selling it; and surveys did indicate that homeowners were ready to default on their mortgages if this were the case [27]. However, according to a report from the Federal Reserve [28], the "median borrower walks away from his home when he is 62 percent underwater" which surprised some observers [29]. Assuming a small downpayment, the cost ratio of finding a replacement at the new lower price, to the cost of staying in the home, is therefore about 2.63, which again is close to $e$.

To summarise, the threshold effect is explained in the quantum model by the contextdependent interference between objective and subjective factors, which leads naturally to the idea of an energy gap. The situation is reminiscent of the photoelectric effect in physics, with the difference that, instead of computing the quantum of energy required to dislodge an electron from an atom, we are computing the quantum of debt required to dislodge a homeowner from their home. In addition, if the idea of a quantum was originally introduced in physics to explain why the ultraviolet catastrophe did not occur in practice, the threshold effect in economics explains the non-occurrence of a less dramatic but still very expensive disaster of a different sort. If those borrowers left underwater by the US 
housing crisis had reacted like rational economic agents and engaged in mass default, then according to an estimate from First American, it would have cost some USD 745 billion, or slightly more than the size of the 2008 bank bailout, to restore the lost equity [30]. The fact this did not happen can be put down to the quantum threshold effect.

\section{The Money Power}

We can therefore define this energy $\Delta E=\hbar \omega / 2$ as the "mental energy" needed to change a person's mind in a financial context. It scales with the frequency $\omega$ which represents a resistance to change. For the supply/demand model discussed above, $\Delta E$ is roughly the energy gap between a buyer and a seller, so adding this energy to the quantum system is equivalent to bringing another party to the negotiating table.

While the above formula defines the social version of a quantum of energy, it still leaves the question of what the individual terms $\hbar$ and $\omega$ represent. In physics, $\hbar$ is a constant with units of action, while $\omega$ is a frequency with units of inverse time. A quantum state with a spread in energy of $\Delta E$ takes a time of at least $\Delta t=\pi \hbar /(2 \Delta E)$ to evolve to a distinguishably different state [31], so it follows that the relevant timescale for a financial decision is $\Delta t=\pi / \omega$.

In order to shed more light on the meaning of these terms in a financial context, we can also compare with another system, which is a debt-based money object (we use the expression "money object" to highlight the fact that money is being treated as a quantum entity [32-34]). Consider a Medieval tally stick which represented a tax obligation. The debtor would be given a stub which recorded the amount owed, while the sovereign retained the stock which recorded the right to collect payment. The stub was therefore an involuntary loan that needed to be paid back, while the stock was valuable in itself and served as a money object. Although stocks did not include an interest rate, they were often sold at a discount, which amounted to an effective interest rate.

If the sovereign had no power, then a tally would be worthless because the debtor would feel no need or desire to pay the debt. The value of the tally therefore reflected the power of the sovereign to produce a preference shift on the part of the debtor. According to the entropic force argument, the energy gap in changing from a default of probability near 1 (in the absence of coercion) to a small probability of default $p$ (high coercion) is $E=\hbar v / 2$ where

$$
v=\omega \log \frac{1}{p}
$$

is a boosted frequency parameter. It is reasonable to assume this frequency scales with the face value of the tally, since a debt can be divided into smaller parts and the energy associated with each part will depend on its size. It also depends on the probability of default $p$, which reflects the soundness of the money supply.

\section{Discussion}

In the picture described above, a money object is therefore a quantum social entity whose frequency or "color" is determined by both its face value and the probability of default. According to one online dictionary [35], "the color of someone's money" means "proof that someone is going to pay you." The quantum economics interpretation makes this expression more exact.

In statistical physics, a frequency $\omega$ is related to the inverse of the Boltzmann time $\tau_{B}=\hbar /\left(k_{B} T\right)$ which is the theoretical order of time needed for an arbitrary nonstationary state to reach thermal equilibrium [36]. In the social version, the timescale then describes (as a first-order model) the characteristic time for which it takes a mental perturbation-or the influence of subjective attractions-to relax or decay by a factor $e$. In other words, it is the time for the threshold effect to reverse itself. If for example a person is resistant to change (large $\omega$ ) then mental perturbations caused by new information will rapidly damp out as the status quo is reasserted (for example the information may be forgotten). A large energy $\gamma=\hbar \omega / 2$ is then required in order to produce a real change. From a modeling 
perspective, since the model is of course just an approximation to the complexities of human behaviour, it may suffice to assume that the financial version of $\hbar$ is constant in a given simulation and allow the frequency $\omega$ to play the role of an adjustable variable which reflects resistance to change.

A common question in quantum economics, discussed also for the quantum oscillator model in [15], is whether a quantum approach is necessary, or if a classical approach can also be used to obtain the same results. As already mentioned, the quantum approach to cognition has many advantages over classical models and in particular it allows preference reversal and related threshold effects to be computed in terms of interference between objective and subjective factors. Comparison with the quantum oscillator model of financial transactions then leads to the expression $\gamma=\hbar \omega / 2$. The quantum approach is therefore required in order to explain the threshold effect in the first place, but in the context of economics it also allows us to find an expression for the quantum of energy in a consistent set of units.

\section{Conclusions}

To recap, we start with a propensity function $P(x)$ which gives the probability of a financial instrument having a particular price $x$. This propensity function is used to derive an entropic force $F(x)$ and can be used to calculate the energy gap between two states. For the case that the initial and final propensities differ by a factor $e$, this becomes $\Delta E=\hbar \omega / 2$.

The formula can be applied to cases where a step shift in propensity is required, as during preference reversal. In the supply/demand model it is the energy needed to shift a potential buyer towards a purchase. For such problems, $\Delta E=\hbar \omega / 2$ can therefore be interpreted as the threshold energy needed to produce a step change or reversal in the system.

In this picture, a transaction or a money transfer is treated as a quantum system with an energy that is determined by a frequency parameter. Applied to the case of a money object such as a tally, the energy is seen to reflect the level of coercion (or more generally persuasion or appeal) implicit in money and the formula implies that the frequency of a money object should scale with both the log probability of default and the face value of the money object. The frequency can therefore be interpreted as defining a kind of resistance to change, as in quantum physics, where it determines how quickly a quantum system equilibrates after a perturbation.

The theory is motivated by the observation that many systems in economics and finance show a threshold effect, which can be modeled using a quantum formalism and this leads inevitably to the idea of a quantum of energy. Of course, this is not the same as saying that "mental energy" is quantized; however, financial transactions are naturally quantized in the sense that there is a single transaction talking place and a payment is a discrete event between two individuals.

Key to the approach is the switch from a framework based on classical logic and expected utility, as in neoclassical economics, to one based on quantum logic and propensity. Many scientists will argue that it is inappropriate to transport ideas such as position, force and energy into the human domain [37]; however, economics is special in that the purpose of the money system is exactly to ascribe a kind of position, known as price, to the fuzzy concept of value and this feature allows us to derive a financial version of energy in terms of an entropic force. The fact that price is just a number, as opposed to something real and immutable, is what introduces the probabilistic uncertainty that makes the quantum approach suitable.

In any case, mainstream economics is already explicitly based on the concept of forces of supply and demand [38], without specifying these in terms of units, or exploring properties such as mass or acceleration. This is possible only because the forces are assumed to be at equilibrium. Applying the idea of entropic forces and quantizing the resulting dynamics, allows us to define these terms more precisely based only on the concepts 
of prices and probabilities. A compelling reason why the quantum formalism can be used in economics is, therefore, that it provides a unified and dimensionally consistent framework for analyzing the dynamics of economic systems. In particular, as seen in this paper, the approach leads to the idea of a quantum of monetary value whose energy exerts a switch-like effect on the human mind, with very real economic consequences.

Funding: This research received no external funding.

Conflicts of Interest: The author declares no conflict of interest.

\section{References}

1. Planck, M. Über das gesetz der energieverteilung im normalspektrum (On the law of distribution of energy in the normal spectrum). Ann. Phys. 1901, 309, 553-563. [CrossRef]

2. Einstein, A. Über einen die Erzeugung und verwandlung des lichtes betreffenden heuristischen gesichtspunkt (On a heuristic viewpoint concerning the production and transformation of light). Ann. Phys. 1905, 17, 132-148. [CrossRef]

3. Busemeyer, J.; Bruza, P. Quantum Models of Cognition and Decision; Cambridge University Press: Cambridge, UK, 2012.

4. Shubik, M. Quantum economics, uncertainty and the optimal grid size. Econ. Lett. 1999, 643, 277-278. [CrossRef]

5. Schaden, M. Quantum finance. Physica A 2002, 3161, 511-538. [CrossRef]

6. Baaquie, B.E. Quantum Finance: Path Integrals and Hamiltonians for Options and Interest Rates; Cambridge University Press: Cambridge, UK, 2007.

7. Haven, E.; Khrennikov, A. Quantum Social Science; Cambridge University Press: Cambridge, UK, 2013.

8. Wendt, A. Quantum Mind and Social Science: Unifying Physical and Social Ontology; Cambridge University Press: Cambridge, UK, 2015.

9. Der Derian, J.; Wendt, A. 'Quantizing international relations': The case for quantum approaches to international theory and security practice. Secur. Dialogue 2020, in press. [CrossRef]

10. Orrell, D. The value of value: A quantum approach to economics, security and international relations. Secur. Dialogue 2020, 51, 482-498. [CrossRef]

11. Planck, M. Scientific Autobiography and Other Papers; New York Philosophical Library: New York, NY, USA, 1949.

12. Khrennikov, A. Ubiquitous Quantum Structure; Springer: Berlin/Heidelberg, Germany, 2010.

13. Khrennikova, P.; Haven, E. Instability of political preferences and the role of mass media: A dynamical representation in a quantum framework. Int. J. Theor. Phys. 2014, 53, 1346-1360. [CrossRef]

14. Khrennikov, A. 'Social Laser': Action amplification by stimulated emission of social energy. Philos. Trans. R. Soc. A 2016, 374, 20150094. [CrossRef]

15. Orrell, D. A quantum model of supply and demand. Physica A 2020, 539, 122928. [CrossRef]

16. Orrell, D. Quantum Economics and Finance: An Applied Mathematics Introduction, 2nd ed.; Panda Ohana: New York, NY, USA, 2021.

17. Sokolov, I.M. Statistical mechanics of entropic forces: Disassembling a toy. Eur. J. Phys. 2010, 31, 1353-1367. [CrossRef]

18. Roos, N. Entropic forces in Brownian motion. Am. J. Phys. 2014, 8212, 1161-1166. [CrossRef]

19. Sarkissian, J. Quantum coupled-wave theory of price formation in financial markets: Price measurement, dynamics and ergodicity. Physica A 2020, in press. [CrossRef]

20. Yukalov, V.I.; Sornette, D. Preference reversal in quantum decision theory. Front. Psychol. 2015, 6, 1538. [CrossRef] [PubMed]

21. Khrennikov, A. Quantum-like modeling of cognition. Front. Phys. 2015, 3, 77. [CrossRef]

22. Yukalov, V.I.; Sornette, D. Quantitative predictions in quantum decision theory. IEEE Trans. Syst. Man Cybern. Syst. 2018, 48, 366-381. [CrossRef]

23. Kahneman, D.; Knetsch, J.L.; Thaler, R. Experimental tests of the endowment effect and the coase theorem. J. Polit. Econ. 1990, 98, 1325-1348. [CrossRef]

24. Güth, W.; Schmittberger, R.; Schwarze, B. An experimental analysis of ultimatum bargaining. J. Econ. Behav. Organ. 1982, 34, 367. [CrossRef]

25. Nowak, M.A.; Page, K.M.; Sigmund, K. Fairness versus reason in the ultimatum game. Science 2000, 289, 1773-1775. [CrossRef]

26. Orrell, D. Quantum-tative finance. Wilmott 2020, 106, 16-23. [CrossRef]

27. Guiso, L.; Sapienza, P.; Zingales, L. The determinants of attitudes toward strategic default on mortgages. J. Financ. 2013, 684, 1473-1515. [CrossRef]

28. Bhutta, N.; Dokko, J.; Shan, H. The depth of negative equity and mortgage default decisions. In Federal Reserve Board; FEDS Working Paper No. 2010-35; 2010; Available online: https:/ / ssrn.com/abstract=1895493 (accessed on 10 March 2021). [CrossRef]

29. White, B.T. Underwater and not walking away: Shame, fear, and the social management of the housing crisis. Wake For. Law Rev. 2010, 45, 971-1023. [CrossRef]

30. Streitfeld, D. No Help in Sight, More Homeowners Walk Away. New York Times, 2 February 2010.

31. Lloyd, S. Ultimate physical limits to computation. Nature 2000, 406, 1047-1054. [CrossRef] [PubMed]

32. Orrell, D. A quantum theory of money and value. Econ. Thought 2016, 52, 19-36. [CrossRef]

33. Orrell, D. Quantum Economics: The New Science of Money; Icon Books: London, UK, 2018. 
34. Orrell, D. Quantum economics. Econ. Thou. 2018, 72, 63-81.

35. The Color of Someone's Money-Definitions and Synonyms. Available online: https://www.macmillandictionary.com/ dictionary/american/the-color-of-someone-s-money (accessed on 6 August 2020).

36. Goldstein, S.; Hara, T.; Tasaki, H. Extremely quick thermalization in a macroscopic quantum system for a typical nonequilibrium subspace. New J. Phys. 2015, 174, 045002. [CrossRef]

37. Pothos, E.M.; Waddup, O.J.; Kouassi, P.; Yearsley, J.M. What is rational and irrational in human decision making. Quantum Rep. 2021, 3, 242-252. [CrossRef]

38. Mankiw, N.G. Principles of Economics, 8th ed.; Cengage Learning: Boston, MA, USA, 2018. 